\title{
Analysis of the Ecological Sensitivity of Pengyang County Based on Key Factors
}

\author{
Shi Yun ${ }^{*}, 1,2$, Geng Sha1, Mi Wenbao ${ }^{1,2}$ and Su Wei ${ }^{1}$ \\ ${ }^{1}$ School of Agriculture, Ningxia University, Yinchuan, Ningxia, 750021, P.R. China \\ ${ }^{2}$ School of Resources and Environment, Ningxia University, Yinchuan, Ningxia, 750021, P.R. China
}

\begin{abstract}
Through selecting key factors such as gradient, exposure, erosive channel, soil erosion and vegetation coverage, the paper establishes the factor index system for the evaluation of the ecological system of Pengyang County, determines the weight of evaluation factors, makes analysis on key ecological factors, sets up the comprehensive sensitivity evaluation model of the ecological system, and obtains the ecological sensitivity grade, area, and spatial distribution of the study area through GIS spatial analysis function, factor superposition method, and analytic hierarchy process. The findings are as follows: the zone extremely sensitive to the ecology covers $82956.47 \mathrm{hm}^{2}$, accounting for $32.98 \%$. Landforms in the area usually include steep slopes with rich vegetation, and high ecological value, erosive channels, and zones affected by erosive channels, all of which are under the key protection; the zone moderately sensitive to the ecology cover $104613.69 \mathrm{hm}^{2}$, accounting for $41.59 \%$. Landforms in the area usually include forestlands of gentle relief and diversified plants. A good many factors should be taken into consideration in the development of the areas; the zone slightly sensitive to the ecology covers $63965.53 \mathrm{hm}^{2}$, accounting for $25.43 \%$. Landforms in the area are usually of low elevation and flat, mainly ordinary greenbelt with singular vegetation, sections with poor vegetation, and farmlands. The area is resistant to human disturbance and able to go through development and construction of the specific intensity. The lands can be developed for a variety of purposes, but attention should be paid to the development intensity.
\end{abstract}

Keywords: Ecological sensitivity, ecological factors, Pengyang County.

\section{INTRODUCTION}

Ecological sensitivity analysis is to evaluate the possibility of a concrete ecological process giving rise to potential ecological and environmental problems under the natural status. Combining ecological sensitivity analysis methods such as ordinary analytic hierarchy process with GIS technology and utilizing GIS spatial data processing and computing capabilities can make the analysis process more concise and the analysis result more accurate, thus offering the reliable data support for related regions [1-3].

Taking Pengyang County located in the loess hilly and gully area as the example, and in the light of the region's research focus and objective conditions, the paper selects factors closely related to the ecological environment and able to produce data, especially natural factors, as key factors for evaluating the ecological sensitivity, including gradient, exposure, erosive channel, soil erosion, and vegetation coverage. The paper determines the weight of evaluation factors through analytic hierarchy process, constructs the comprehensive sensitivity evaluation model of the ecological system of Pengyang County according to GIS spatial analysis, and finally finds out the spatial distribution of different ecological sensitive areas, thus offering the basis for understanding the region's ecological environment status and making scientific ecological planning.

*Address correspondence to this author at the School of Agriculture, Ningxia University, Yinchuan, Ningxia, 750021, P.R. China;

Tel: 13995077221; E-mail: shiysky@163.com

\section{THE STUDY AREA}

Pengyang County is located at the southeast of Ningxia Hui Autonomous Region, with east longitude being $106^{\circ}$ $32^{\prime}-106^{\circ} 58^{\prime}$, north latitude $35^{\circ} \quad 41^{\prime}-36^{\circ} 17^{\prime}$, and elevation $1,248-2,483 \mathrm{~m}$. The county is of the typical landform of the second sub-region of the loess hilly and gully area, mainly loess hills. The topography is shattered and disconnected, with steep hills and crisscross gullies [4]. The landform is composed of ridge, tableland, valley, and gully. Gully density reaches $2.4 \mathrm{~km} / \mathrm{km}^{2}$. Therefore, Pengyang County is one of the counties with the most serious water and soil erosion in Ningxia and also the key water and soil conservation zone in China.

\section{STUDY FOUNDATION}

\subsection{Data Source and Processing}

The paper takes 1:50,000 present land-use map (2010), 1:100,000 topographic map, soil erosion data, and statistical yearbook of Pengyang County as basic data for the study. First of all, it makes use of ArcGIS10.0 software to geometrically proofread the present land-use map, then rectifies the images to obtain the landscape data of the study area, and converts vector data into raster data. Raster data are defined as $25 \mathrm{~m} \times 25 \mathrm{~m}$. Via the analytic function of ArcGIS software, the paper produces the gradient and exposure raster figure with 1:100, 000 elevation model as data source. Raster data are defined as $30 \mathrm{~m} \times 30 \mathrm{~m}$. 


\subsection{Methodology}

The research is to analyze the ecological sensitivity of the study area on the basis of GIS spatial analysis and weighted stacking method. In the light of original data collected and evaluation goal, the paper analyzes and selects key single factors for evaluating the ecological sensitivity of the study area, grades each single factor, draws out the single factor rating map [5-7], and determines the weight of each single factor through AHP. On the basis of DEM and via D8 algorithm, the paper automatically retrieves data of erosive channel, gradient, exposure and soil erosion in the northwestern loess plateau region, makes use of GIS spatial analysis methods such as raster data analysis, vector data analysis, and 3D spatial analysis to stack weights of key factors, and finally acquires the stacking result rating, namely the ecological sensitivity rating map, for the purposes of analyzing the spatial distribution of the ecological sensitivity of Pengyang County and further proposing related measures of ecological planning [8-11].

\subsection{Selection of Key Factors for the Evaluation of Ecological Sensitivity}

Ecological sensitivity analysis is to evaluate the possibility of a concrete ecological process giving rise to potential ecological and environmental problems under the natural status. The most serious ecological problem of Pengyang County finds expression in water and soil erosion and crisscross gullies. According to the implication of ecological sensitivity analysis, the paper selects related factors in terms of natural ecology, such as geology, landform, gradient, soil, hydrology, vegetation, biological diversity, climate, etc., and then distinguishes those resulting in water and soil erosion from the factors. Key factors for the evaluation of the ecological sensitivity of Pengyang County include gradient, exposure, erosive channel, soil erosion, vegetation coverage, etc.

In the light of the rating of the ecological sensitivity, the study area is divided into three rating zones in the evaluation, namely strong ecological sensitive zone, medium ecological sensitive zone, and slight ecological sensitive zone. In the light of the ecological sensitivity analysis, related ecological factors fall into different sensitivity value from $0-10 .$. Rating scale of related ecological sensitive factors is shown as Table $\mathbf{1}$.

\section{ANALYSIS ON GRADIENT FACTOR}

Pengyang County is of varied landforms such as valley, ridge, and flat ground. Gradient factor is determined according to the provision of the Law of the People's Republic of China on Water and Soil Conservation: Reclamation of hillsides with a slope of over $25^{\circ}$ for cultivation of crops shall be prohibited [13]. In addition, as a demonstrative county of returning farmland to forest nationwide, Pengyang County focuses on returning farmland in mountainous areas of gradient being above $15^{\circ}$ and dry farmland in serious windy and sandy areas to forest and grassland with small watershed as the unit for scale treatment. The county combines basic construction of farmlands with structure adjustment of agriculture to return and treat the farmland one by one. Therefore, farmlands in mountainous areas of gradient being above $15^{\circ}$ are also covered in the scope of grain for green. Distribution of different gradients of Pengyang County is shown as the figure, and areas shown as Table $\mathbf{2}$.

\subsection{Exposure Factor Analysis}

As an important part of the microclimate, exposure has a bearing on the variety and distribution of vegetation and animals, lighting of buildings, and utilization efficiency of energies. As far as construction is concerned, south slope of a hill is superior to the north slope. The most favorable exposure is the south-by-east slope, where plants can receive sufficient lighting and ventilation, thus being favorable for their growth. Hence, exposure factor can be determined. Pengyang County's flat ground covers an area of 27,538.26 $\mathrm{hm}^{2}$, mainly valley areas and southwest water areas as well as the surrounding areas, accounting for $10.95 \%$ of the total; true north region covers an area of $24,309.68 \mathrm{hm}^{2}$, accounting for $9.66 \%$ of the total; northeast and northwest regions cover an area of $54,393.65 \mathrm{hm}^{2}$, accounting for $21.62 \%$ of the total; true west and true east regions cover an area of $49,985.72 \mathrm{hm}^{2}$, accounting for $19.87 \%$ of the total; southeast and southwest regions cover an area of 59,187.60 $\mathrm{hm}^{2}$, accounting for $23.53 \%$ of the total; and true south region covers an area of $36,120.78 \mathrm{hm}^{2}$, accounting for $14.36 \%$ of the total.

\subsection{Channel Factor Analysis}

Channel erosion is the major reason for water and soil loss in loess plateau. Since loess is vulnerable to the erosion of flowing water, there form a mass of widely distributed erosive channels [12]. Channel properties are also major factors affecting channel erosion, such as channel length, elevation difference, and vertical gradient, of which elevation difference can generate the biggest influence. In general conditions, the bigger the elevation difference is, the bigger the surface flow speed is, and the more serious channel erosion is. Therefore, the study decides on the ecological sensitivity on the basis of related channel properties, extracting catchment area of erosive channels with DEM as data source and processing properties to calculate elevation difference and vertical gradient. Channel vertical gradient is the key factor considered in the treatment of erosive channels. Generally speaking, the bigger the vertical gradient is, the more serious the erosion is.

According to the above findings, the paper carries out statistical analysis, and obtains related data such as channel length, elevation difference, and vertical gradient, and also rating distribution of different drainage areas in the light of catchment area of channels. Channel vertical gradient is the key factor considered in the treatment of erosive channels. Generally speaking, the bigger the vertical gradient is, the more serious the erosion is. Vertical gradients of different channels and the rating distribution of different drainage areas of Pengyang County are shown as Table $\mathbf{3}$.

\subsection{Soil Erosive Factor Analysis}

Soil erosion data are divided into five grades of minor, slight, medium, intense, extremely intense-severe in terms of 
Table 1. Rating scales of ecological sensitive factors.

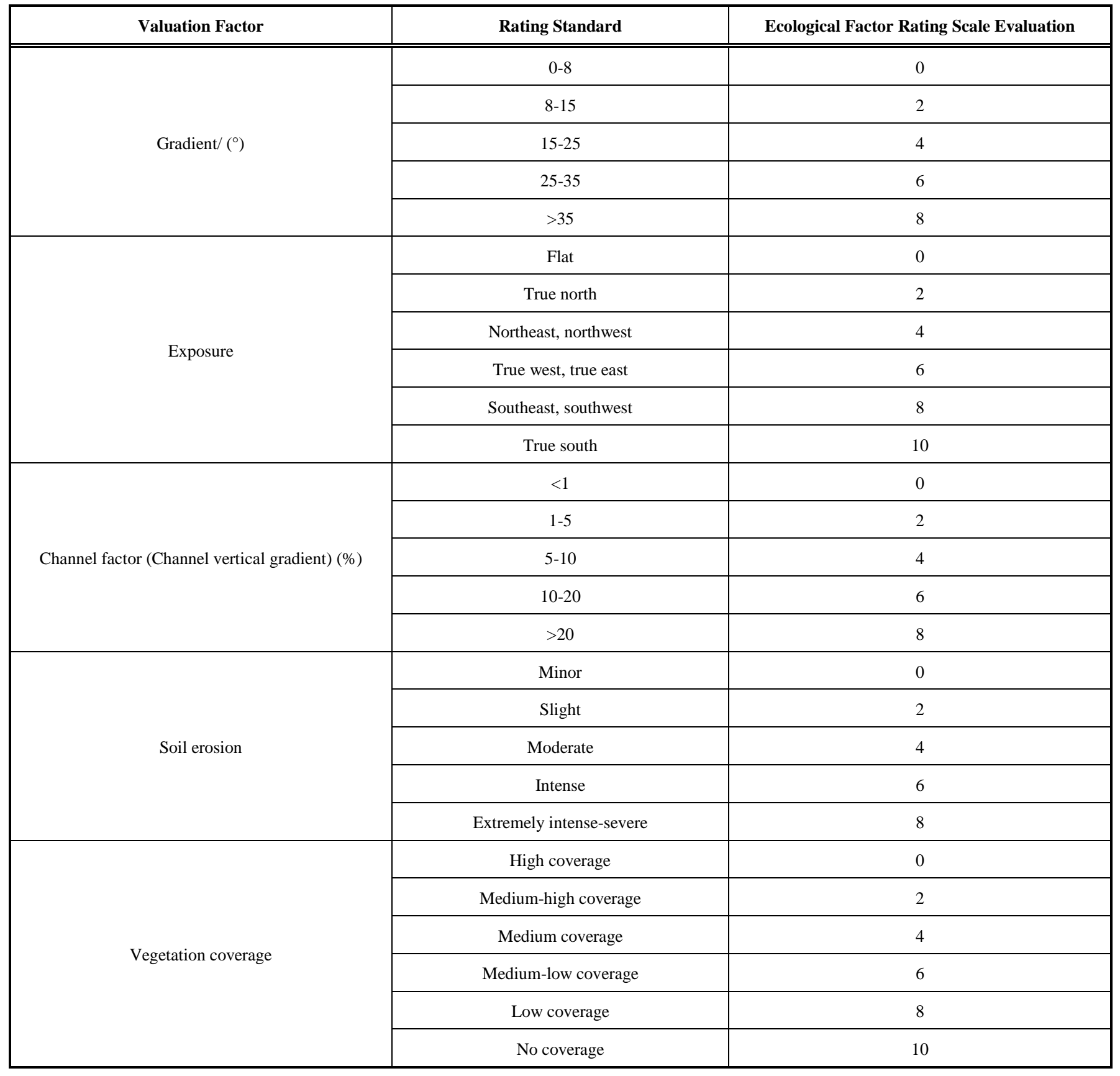

Table 2. Distribution of gradients of pengyang county $\left(\mathrm{hm}^{2}\right)$.

\begin{tabular}{|c|c|c|c|c|}
\hline Gradient rating & $\mathbf{0 - 8}$ & $\mathbf{8 - 1 5}^{\circ}$ & $\mathbf{1 5 - 2 5}^{\circ}$ & $\mathbf{2 5 - 3 5}^{\circ}$ \\
\hline \hline Area & 81953.25 & 78274.32 & 70181.90 & 18789.15 \\
\hline
\end{tabular}

erosion intensity, and area distribution of different soil erosion intensities is shown as Table 4.

\subsection{Vegetation Coverage Factor Analysis}

Vegetation is the most important part of biological resources and one of the most important factors affecting the ecological sensitivity. Vegetation resources can be upgraded continuously in natural conditions or artificial maintenance; on the contrary, they may degrade or disappear in harsh environmental conditions or under artificial destruction or unreasonable utilization, and the process is sometimes irreversible. Vegetation resource distribution varies from region to region, so do their species composition and 
structural features. Areas of vegetation coverage are shown as Table $\mathbf{5}$.

\section{RESULTS AND ANALYSIS}

\subsection{Weight Determination}

Using AHP, based on 5 experts to build judgment matrix of soil and water conservation, calculate the Exposure, Gradient and other factors Weight value, which are shown as Table $\mathbf{6}$.
$\lambda \max =6.139566$;
IC. I=0. 027 913;
$\mathrm{RC} . \mathrm{R}=0.031$.

According to the calculation results above, if $\mathrm{RC} . \mathrm{R}<0.1$, the matrix is deemed to have the satisfactory consistency. Otherwise, it should be adjusted. If $\mathrm{RC} . \mathrm{R}<0$. 1, it is unnecessary to adjust the matrix.

\subsection{The Evaluation Model}

Transform the ecological sensitivity single factor layer into raster data format, then apply multiple factor model of weighted summation to do superposition calculation, generate the ecological sensitivity evaluation map of Pengyang county. The specific calculation formula is:

$S=\sum_{i=1}^{\mathrm{n}} w_{i} p_{i}$

where,

$S$--- the ecological sensitivity level of the land use;

W ---the weight of the $\mathrm{i}$ evaluation factor;

$\mathrm{p}_{i}$---the numerical value of the $\mathrm{i}$ ecological sensitivity evaluation factor;

$\mathrm{n}$----the number of evaluation factors, $n=1,2, \ldots, 5$.

Table 3. Relations between channel vertical gradient and drainage area $\left(\mathrm{hm}^{2}\right)$.

\begin{tabular}{|c|c|c|}
\hline Rating & Channel vertical gradient(\%) & Drainage area \\
\hline \hline 1 & $<1$ & $155,500.99$ \\
\hline 2 & $1-5$ & $12,837.24$ \\
\hline 3 & $5-10$ & $49,058.79$ \\
\hline 4 & $10-20$ & $31,524.53$ \\
\hline 5 & $>20$ & $2,614.14$ \\
\hline
\end{tabular}

Table 4. Area of different soil erosion intensities of pengyang county $\left(\mathrm{hm}^{2}\right)$.

\begin{tabular}{|c|c|c|c|c|c|}
\hline Erosion intensity & Minor & Slight & Moderate & Intense & Extremely intense-severe \\
\hline \hline Area & $34,855.43$ & $42,679.39$ & $80,245.59$ & $59,253.75$ & $34,501.53$ \\
\hline
\end{tabular}

Table 5. Vegetation coverage $\left(\mathrm{hm}^{2}\right)$.

\begin{tabular}{|c|c|c|c|c|c|c|}
\hline Vegetation coverage & High coverage & Medium-high coverage & Medium coverage & Medium-low coverage & Low coverage & No coverage \\
\hline Area & $7,366.82$ & $30,089.13$ & $51,867.33$ & $94,111.97$ & $64,136.3$ & $3,964.14$ \\
\hline
\end{tabular}

Table 6. Weight analysis.

\begin{tabular}{|c|c|c|c|c|c|c|}
\hline Evaluation factor & \multicolumn{5}{|c|}{ Score of ecological sensitivity } & Weight value \\
\hline \hline & Exposure & Gradient & Erosive channel & Soil erosion & egetation & $1 / 4$ \\
\hline Exposure & 1 & $1 / 2$ & $1 / 3$ & $1 / 3$ & 1 & $1 / 2$ \\
\hline Gradient & 2 & 1 & 1 & 1 & 1 & 0.077 \\
\hline Erosive channel & 3 & 1 & 1 & 1 & 1 & 0.187 \\
\hline Soil erosion & 3 & 1 & 1 & 1 & 1 & 0.187 \\
\hline Vegetation & 4 & 2 & & & 0.447 \\
\hline
\end{tabular}


Table 7. Areas \& proportions of different ecological sensitive zones of pengyang county.

\begin{tabular}{|c|c|c|}
\hline Sensitivity rating & Area/ $\mathrm{hm}^{2}$ & Proportion/\% \\
\hline \hline Strong sensitive zone & $82,956.47$ & 32.98 \\
\hline Medium sensitive zone & $104,613.69$ & 41.59 \\
\hline Slight sensitive zone & $63,965.53$ & 25.43 \\
\hline Total & $251,535.69$ & 100.00 \\
\hline
\end{tabular}

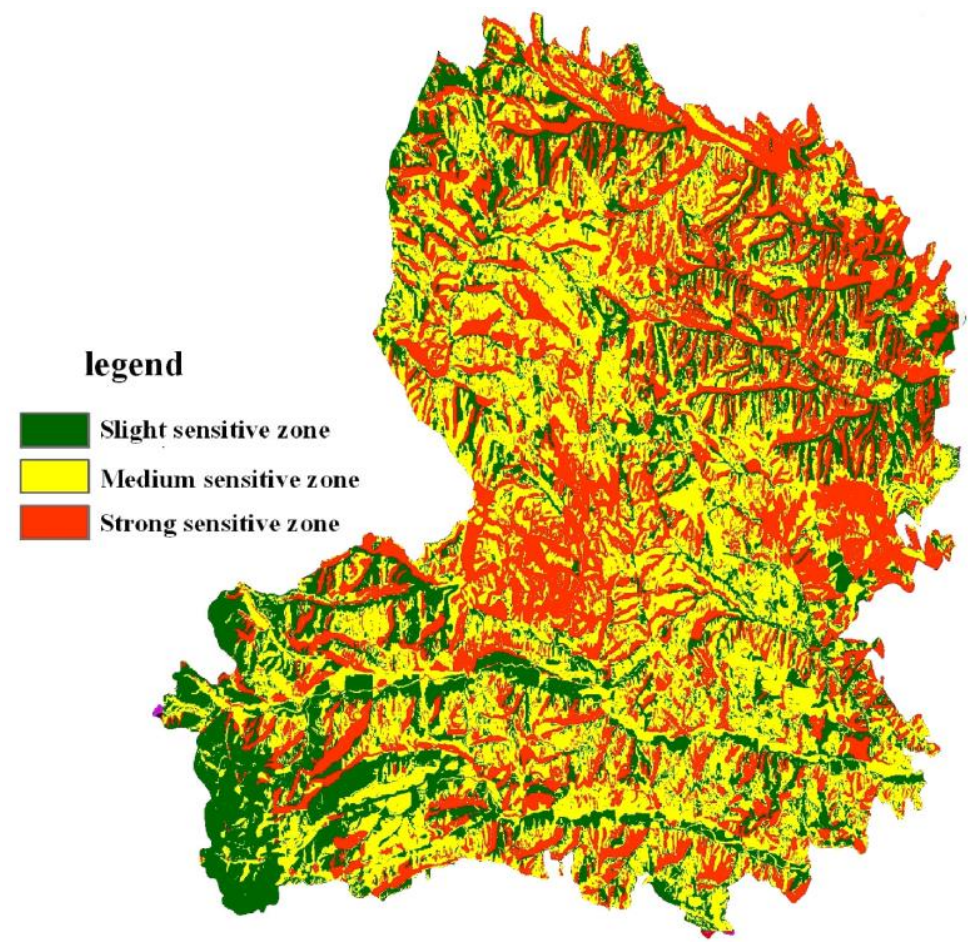

Fig. (1). The corresponding areas of different sensitive zones.

\subsection{Multi-factor Weighted Stacking Ecological Sensitivity Analysis}

Gradient, exposure, small watershed, soil erosion and vegetation are taken as evaluation factors in the ecological sensitivity analysis. After superposition of multiple factor analysis, final score according to the natural discontinuities classification method from 0 to $3.14,3.14$ and $4.87,4.87$ and 8.84 in Pengyang county was divided into slight sensitive zone, medium sensitive zone and strong sensitive zone, at the same time get the ecological sensitivity rating map of Pengyang County is then obtained through weight stacking, shown as Fig. (1). The corresponding areas of different sensitive zones are shown as Table 7.

As shown in the table7, the strong sensitive zone covers an area of $82,956.47 \mathrm{hm}^{2}$, accounting for $32.98 \%$ of the total, mainly distributed in the middle and north parts of of Pengyang County, involving loess ruin areas of Wangwa Town, Baiyang Town, Xiaocha Town, and Mengyuan Town; the medium sensitive zone covers an area of $104,613.69 \mathrm{hm}^{2}$, accounting for $41.59 \%$ of the total, mainly distributed at the south, east and southeast parts of the county; and the slight sensitive zone covers an area of $63,965.53 \mathrm{hm}^{2}$, accounting for $25.43 \%$ of the total, mainly distributed in the southwest part of Pengyang County, and valleys of Ruhe River and Honghe River.

\section{DISCUSSION}

With factor weighted stacking method and fuzzy evaluation method as the theoretical supports, the paper establishes the ecological factor evaluation index system, determines the evaluation standard, and concludes that the multi-factor weighted stacking is the commonly used method for evaluating the ecological sensitivity. By selecting key factors, the research, different from previous ones, focuses on the gradient and soil erosion-especially channel factorrelated to the evaluation of the ecological sensitivity of the study area. Through extracting data of erosive channel and small watersheds, and taking channel vertical gradient as referential index, the paper divides small watersheds into different ratings in terms of the possibility of water and soil loss, thus offering the scientific basis for evaluating the ecological sensitivity of the study area and obtaining the rating spatial distribution of the ecological sensitivity of 
Pengyang County. After the analysis, the paper comes to the following conclusions:

1. The strong sensitive zone of the study area covers $82,956.47 \mathrm{hm}^{2}$, accounting for $32.98 \%$. Landforms in the area usually include steep slopes with rich vegetation, and high ecological value, erosive channels, and zones affected by erosive channels. The zone is extremely sensitive to development and construction. Any disturbance or destruction to the zone would affect the complex ecological system of the whole region. Therefore, the strong sensitive zone is under the key protection.

2. The medium sensitive zone covers $104,613.69 \mathrm{hm}^{2}$, accounting for $41.59 \%$ of the total. Landforms in the area usually include forestlands of gentle relief and diversified plants. The lands are highly sensitive to human activities, and the ecology, which it is hard to restore, plays the important role in maintaining the function and environment of the strong sensitive zone. It should be considered whether it is feasible to develop and utilize the medium sensitive zone.

3. The slight sensitive zone covers $63,965.53 \mathrm{hm}^{2}$, accounting for $25.43 \%$. Landforms in the area are usually low and flat, mainly rivers and forests with singular vegetation, areas with poor vegetation, and farmlands. The areas are resistant to human disturbance and able to go through development and construction of the specific intensity, and the lands can be developed for a variety of purposes. However, serious disturbance may lead to water and soil loss as well as related natural disasters, and the ecological restoration is slow.

\section{ABOUT THE AUTHORS}

\section{First Author}

Shi Yun, Associate professor, studying for $\mathrm{PhDs}$ in Ningxia University. The author's major is Grass science.

\section{Second Author}

Geng Sha, studying for master degree in Ningxia University. The author's major is GIS.

\section{Third Author}

Mi Wenbao, Professor of Ningxia University. The author's major is Human geography.

\section{CONFLICT OF INTEREST}

The authors confirm that this article content has no conflict of interest.

\section{ACKNOWLEDGEMENTS}

This work was financially supported by the Natural Science Foundation of China (41161081).

\section{REFERENCES}

[1] Z. Wu, M. Liu, Z. Wang, GIS-based Sensitivity Evaluation of Water and Soil Loss in Anshan City, Liaoning, 2009.

[2] Z. Ouyang, X. Wang, H. Miao, "China's eco-environmental sensitivity and its spatial heterogeneity", Acta Ecologica Sinica, vol. 20, pp. 9-12, 2000

[3] J. Pan, X. Dong, "GIS-based assessment and division on ecoenvironmental sensitivity in the heihe river basin", Journal of Natural Resources. vol. 21, pp. 267-272, 2006

[4] Compiling Committee of Annals of Pengyang County, Annals of Pengyang County, Yinchuan, 1996

[5] X. Tao, J. Zhang, Y. Wang, "Eco-sensitivity and its spatial distribution in hangzhou", Journal of Hangzhou University, vol. 32, pp. 27-30, 2006

[6] J. Mo, Y. Lu, L. Wei, "Evaluation \& analysis on urban ecosensitivity in nanning based on GIS", Geomatics World, vol. 1, pp. 33-38, 2007

[7] J. Hong, Y. Song, "application research of analytic hierarchy process (AHP) in water environmental planning", Environmental Science \& Technology, vol. 1, pp. 35-35, 2000

[8] J. Liu, "Discussion on the method of making slope classification map with DEM in ArcGIS", Geomatics \& Spatial Information Technology, vol. 34, pp. 140-141, 2011

[9] B. Ren, H. Tian, "Study on indicators and methods with regards to national eroded gully survey", Yangtze River, vol. 41, pp. 103-105, 2010

[10] J. Ren, A. Ding, W. Liu, "Study and realization of automatic extraction method for gully erosions in northwest loess plateau area", Journal of Gansu Sciences, vol. 24, pp. 16-19, 2012

[11] H. Feng, Y. Shang, X. Liu, J. Ma, "Conversion method of extracting ground gradient through different resolution dem in lanzhou", Journal of Lanzhou University (Natural Sciences), vol. 1, pp. 80-84, 2010

[12] Y. Feng, L. Yuexiang, Study on Returning Farmland to Forest Project in Ningxia, Yinchuan, pp. 13-19, 2012.

[13] L. Jinzhao,G. Qingxi,G. Jianping, " DEM based automated extraction system of gully in the loess upland gully area", Journal of Northwest Forestry University, vol. 24, pp. 220-223,2009. 